\title{
Disruption of the Choroidoretinal Interface by Toxoplasma
}

\author{
KHALID F. TABBARA \\ Riyadh, Saudi Arabia
}

\begin{abstract}
Summary
Toxoplasmosis is a common zoonotic infection of the retina caused by Toxoplasma gondii. The disease causes severe damage that affects the retina and subjacent choroid. Invasion of the retinal cells by the parasite leads to the major damage seen in the retina. Subsequent reactions to the invasion by toxoplasma leads to the influx of inflammatory cells. Although hypersensitivity reactions have been described to both toxoplasma and to retinal autoantigens, the major disruption of the choroidoretinal interface is probably secondary to the tissue invasion by the parasite. Patients with AIDS may show extensive necrosis of the retina in the absence of inflammatory cells.

Healing of the active lesion leads to scar formation. Toxoplasma retinochoroiditis may be pigmented, nonpigmented or punched-out. Vascular anastomoses between the retina and choroid, retinal and subretinal neovascularization may occur in the aftermath of choroidoretinal interface disruption.
\end{abstract}

Pathogenic and opportunistic infections may afflict intraocular structures. The retina and choroid are frequently the target of insult by a variety of infectious agents including: viruses, bacteria, fungi and parasites. This leads in some instances to the disruption of the choroido-retinal interface. The degree of disruption depends on the tissue involved, the biologic characteristics of the organism, the interaction between the organism and the host cell, the tissue damage caused by the organism and the inflammatory response. The repair of the interface may lead to the formation of a disorganised scar. Certain infections cause a predominant retinitis, some lead to choroiditis, yet other infections may cause both. Viruses such as the herpes group (CMV, Herpes simplex, Herpes zoster, Epstein-Barr virus) and rubella and rubeola viruses cause retinitis. The obligate intracellular parasite
Toxoplasma may lead to a necrotising retinitis and the predominant target of injury in such an infection is the retina, but the subjacent choroid may be involved by the inflammatory process and the choroidoretinal interface may thus be affected. On the other hand, the choroid may be primarily and initially involved in endogenous bacterial and fungal infections such as tuberculosis brucellosis, syphilis, borreliosis, cryptococcosis, and histoplasmosis. The inflammatory processes in such infections can spread quickly, however, to the overlying retina disrupting the choroidoretinal interface.

The main objective of this paper is to study the mechanisms involved in the disruption of the choroidoretinal interface and the sequelae of such disruption in ocular toxoplasmosis.

Toxoplasma gondii: The term 'toxo' is derived from the Greek word toxon or arc

From the Department of Ophthalmology, College of Medicine, King Saud University, Riyadh, Saudi Arabia. This study was supported in part by a grant from the Saudi Eye Foundation, Jeddah, Saudi Arabia

Correspondence to: Professor K Tabbara, Department of Ophthalmology, College of Medicine, King Saud University, P.O. Box 55307, Riyadh 11534, Saudi Arabia. 


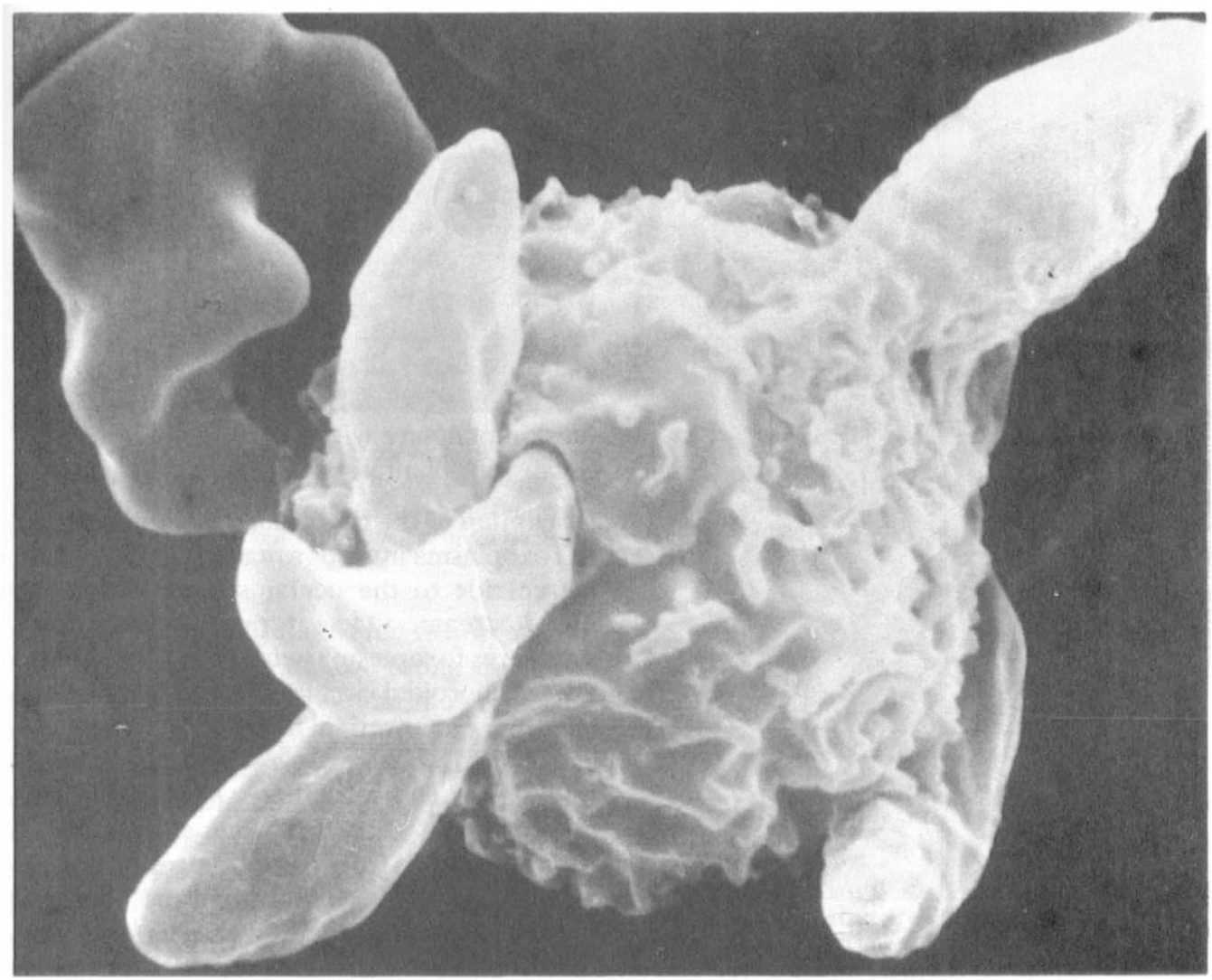

Fig. 1. Scanning electron microscopic picture showing several toxoplasmas attempting to penetrate a macrophage. (Courtesy M L Chiappino and B Nichols)

because of the crescentic shape of the parasite. Toxoplasma is an obligate intracellular protozoon that causes toxoplasmosis in man and animals. The disease is widespread in nature and is considered to be the most common cause of infection of the retina. Infestation with the parasite Toxoplasma gondii is commonly encountered in large segments of the population of both the temperate and tropical zones of the world. It is clear from toxoplasma antibody testing of ostensibly normal individuals that most of these infections are asymptomatic. ${ }^{1}$ Since toxoplasma is an obligate intracellular parasite, its survival depends on two major functions namely locomotion and penetration of the host cell. Both of these functions are achieved by highly differentiated subcellular structures. Under microscopy movements of live toxoplasma may be observed. The organism has a rostral end known as the conoid and possesses no fla- gellum. The movement of the parasite depends on intracellular and pellicular microtubular structures. The organism can twist, wiggle, rotate and glide. Spiraling, undulating and boring motility has been observed indicating several mechanisms operating simultaneously or sequentially., ${ }^{2,3}$ The conoid moves independently. It may extend, retract, tilt, or rotate. ${ }^{2,3}$ When a suitable host cell is encountered, the conoid extends probing the surface of the cell and thrusting forward and inward causing disruption of the cell membrane by the mechanical force of the conoid and by the release of lytic enzymes from the rhoptries (cellular substructures found in the conoid of toxoplasma). ${ }^{3,4}$

The penetration of the host cell by toxoplasma takes 30 seconds. ${ }^{4}$ The entry into the host cell is a crucial step in the survival of the organism. The conoid has three ring structures: anterior preconoidal ring, the posterior 


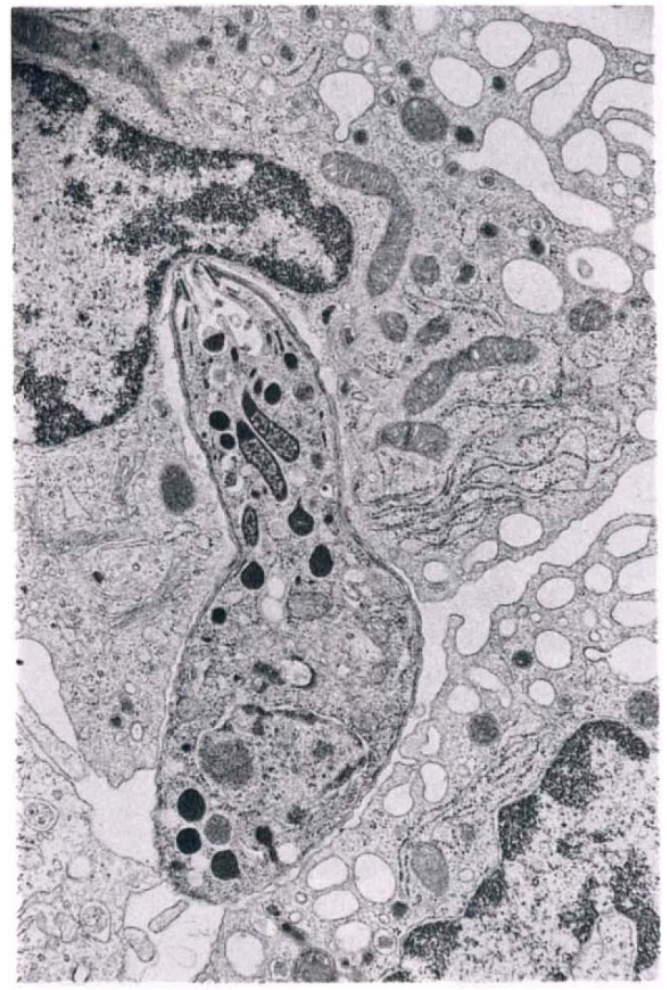

Fig. 2. Transmission electron microscopic photograph of a toxoplasma organism disrupting a mouse cell membrane and penetrating the cell with its conoid (27, $150 x$ ). The picture was taken 60 seconds after injection of toxoplasmas into the mouse peritoneal cavity. (Glutaraldehyde fixation). (From reference 5).

preconoidal ring, and the polar ring. ${ }^{2}$ The pellicular microtubules are attached to the polar ring. contractile elements have been demon-

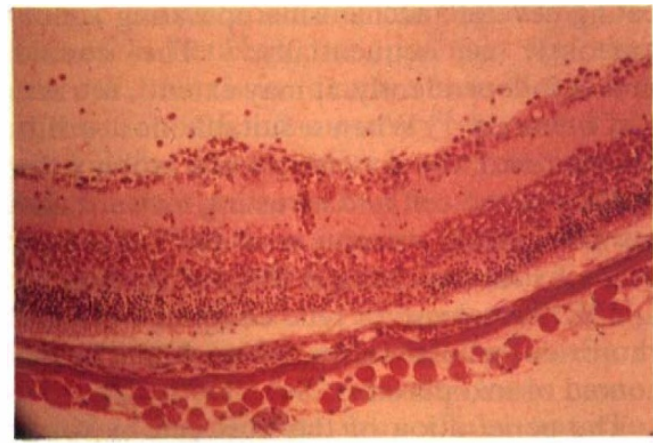

Fig. 3. $H \& E$ stained section of a mouse retina two weeks after intraperitoneal injection of Toxoplasma gondii (RH stain). Showing a toxoplasma cyst in the inner layers of the retina $(40 \mathrm{x})$. strated in toxoplasma and cytocholosin D (known to interfere with microfilament function) can inhibit the host cell penetration by toxoplasma. ${ }^{5,6}$

Man can be infected by toxoplasma by the inhalation of oocysts containing sporozoites, or by ingestion of cysts containing bradyzoites in undercooked meat, or by transplacental transmission of tachyzoites in an acutely infected mother. ${ }^{1,7}$ Less common modes of transmission of tachyzoites include the ingestion of infected milk, transcutaneous accidental skin puncture with infected needles, or by organ transplantation.

\section{Disruption of the choroidoretinal interface:}

Toxoplasma invades a macrophage and gets a free ride to the ocular structures via the blood stream. Once it reaches the ocular structures toxoplasma gets access to the retina via the choroidal circulation or the retinal circulation. Outer retinal foci of infection can be established when toxoplasma reaches the retina via the choriocapillaris, while inner retinal infections occur following entry into the retinal host cells from the retinal terminal capillaries. I injected 1000 organisms of Toxoplasma gondii (Beverley Strain) into the peritoneal cavities of 11 Swiss-Webster mice. The mice were sacrificed two weeks later and their eyes were enucleated and cut in half. One half was fixed and stained for histopathologic evaluation, and the retina and choroid of the other half were minced and ground with Hank's BSS and injected into the peritoneal cavities of healthy Swiss-Webster mice to recover Toxoplasma gondii. In nine (81\%) out of the 11 mice I observed Toxoplasma

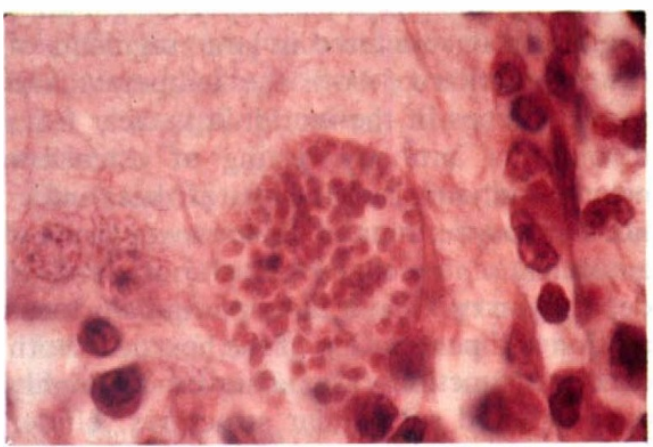

Fig. 4. Toxoplasma cyst at high magnification (400 x) (same as in Fig. 3) 


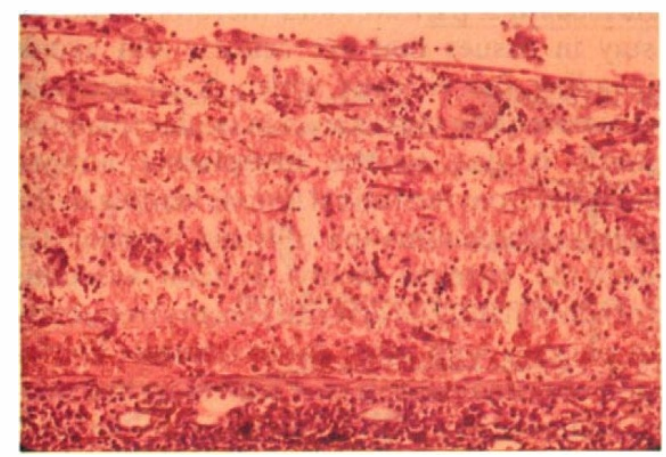

Fig. 5. $H \& E$ stained section of the retina of a patient who had ocular toxoplasmosis. The eye was blind and painful with secondary glaucoma. Note diffuse necrosis of the retina and marked mononuclear inflammatory infiltration of the subjacent choroid.

cysts in the retina or recovered the organisms in mice injected with minced retina and choroid. This suggests that toxoplasma organisms can reach the ocular structures efficiently following systemic infection. In the rabbit model of ocular toxoplasmosis, toxoplasma organisms were injected into the suprachoroidal space with a $30 \mathrm{~g}$ needle attached to a microliter syringe. ${ }^{8}$ The retinal lesions appeared as early as 48 hours after the injection. The initial lesions of the outer retina appeared as localised round foci of retinal pigment epitheliitis which progressed into a focus of retinochoroiditis. 8.9

In subhuman primates we introduced toxoplasma organisms with a blunt $30 \mathrm{~g}$ needle through the pars plana, and the organisms were deposited on to the retina. Again a focus

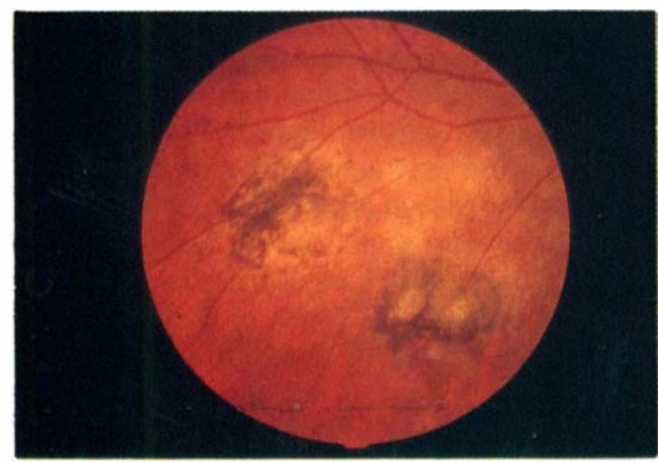

Fig. 6. Toxoplasmic retinochoroiditis in a patient who had two previous attacks of retinochoroiditis. Note the active necrotic retinal lesion is adjacent to old healed scars. of inner retinitis was observed within 48-72 hours after the introduction of the organisms, and this was followed by the appearance of a soft white infiltrate. ${ }^{10}$

The ocular disease in humans may be congenital or acquired ranging in its manifestation from a small peripheral focus of retinochoroiditis to a large posterior fulminating granuloma with necrotising retinitis and choroiditis. It is generally stated that congenital toxoplasmosis produces bilateral ocular disease, and that acquired disease produces monocular lesions. Exceptions, however, can be found to both rules. In general congenital ocular toxoplasmosis lesions are multiple, but single isolated lesions are observed. In congenital infections, the most serious damage occurs during the period of acute infection in utero. Once the lesion becomes inactive, it may later produce symptoms at completely unpredictable times.

Unlike infections of other tissues, toxoplasmic infection leads to severe necrosis and damage of the retina and choroid eventuating in disruption of the choroidoretinal interface.

Following a primary infection with Toxoplasma gondii, the organism may stay viable within cysts in various tissues including brain, eye, or muscle throughout life. Recurrence and recrudescence of the disease may occur following immunologic suppression.

The ocular manifestations of toxoplasmosis consist of a localised area of inflammation. The subjacent choroid may also eventually become affected by the inflammatory cells. The retinal lesions tend to show variations in manifestations. The lesion may be a single focus of necrotising retinitis involving the

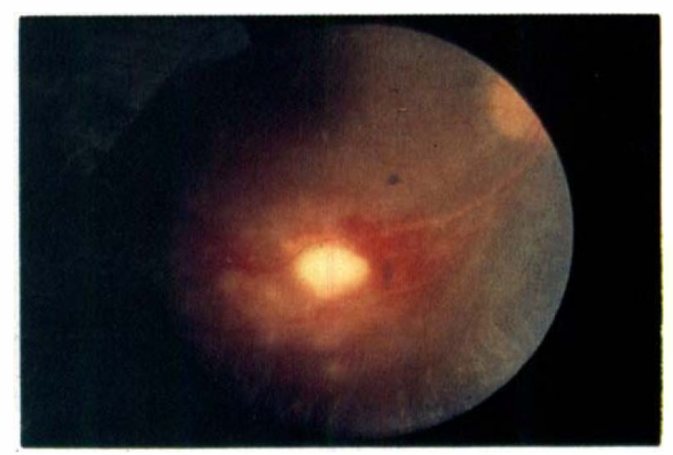

Fig. 7. Localised toxoplasmic retinochoroiditis with retinal branch arteritis. 


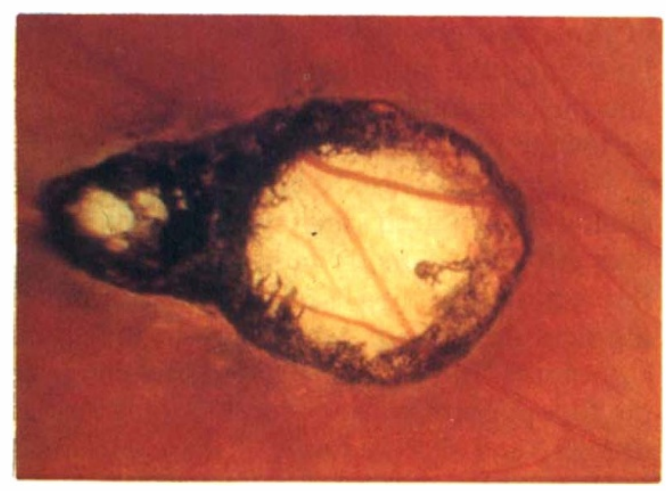

Fig. 8. A healed retinochoroiditic scar showing the aftermath of retinochoroiditis surrounded by pigmentation.

inner retinal layer in the company of an old retinochoroiditic scar. Other lesions may be punctate and in the outer layers of the retina involving the retinal pigment epithelium with satellite lesions. Fundus lesions of toxoplasmic outer retinitis may appear as punctate deep retinal white spots as described by Doft and Gass. ${ }^{11}$ Fluorescein angiography shows early blockage and late staining. Another mode of presentation is a large granuloma with severe necrotising retinitis, choroiditis, and vitritis. The retina, however, in all those variations sustains the primary insult and major damage. Toxoplasma lesions of the inner retinal layers appear as soft fluffy white infiltrates surrounded by retinal oedema. Toxoplasma organism tends to avoid the choroid and proliferates in the relatively immunologically compromised tissue, the retina. On the other hand, the organism is an obligate

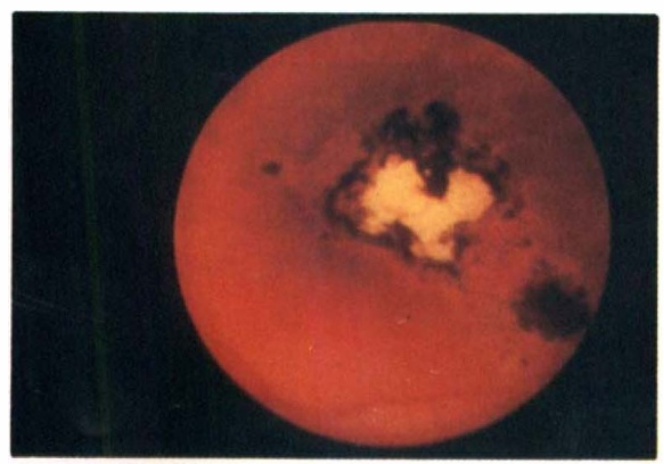

Fig. 9. A punched-out toxoplasmic retinochoroiditic scar surrounded by dark pigmentation in a 17-year-old black patient. intracellular parasite, and therefore tends to stay in tissues that are cellular and avoids invading the vitreous which is an acellular structure. The vitritis seen in ocular toxoplasmosis is due to the toxoplasma antigen released from the retinal lesions leading to the ingress of inflammatory cells and lymphocytes into the vitreous cavity. Retinal vasculitis (predominantly an arteritis) occurs frequently in patients with inner layer retinitis. Occlusion of retinal vessels may lead to retinal neovascularisation. In experimental animals Rollins et $a l^{12}$ have found that toxoplasma antibodies and toxoplasma antigens may be detected in the vitreous of rabbits having toxoplasmic retinochoroiditis. The toxoplasma antigen is detected at the height of the activity of the disease and antibodies can be detected at six weeks after the onset of retinochoroiditis. ${ }^{11}$ Antigens released into the vitreous cavity from contiguous inflammatory lesions of the retina may persist for weeks or months after the initial insult. This may be responsible for the perpetuation of chronic inflammatory responses in the vitreous long after the long initial retinal lesions of ocular toxoplasmosis appear to have subsided.

The severe damage that affects the retina and subjacent choroid of individuals suffering from toxoplasmic retinochoroiditis can be attributed to several factors. The primary insult to the retinal cells is probably the most important of these. Subsequent inflammatory reaction at the choroidoretinal interface follows the parasite invasion and may attract large numbers of macrophages, lymphocytes, plasma cells and neutrophils which helps to

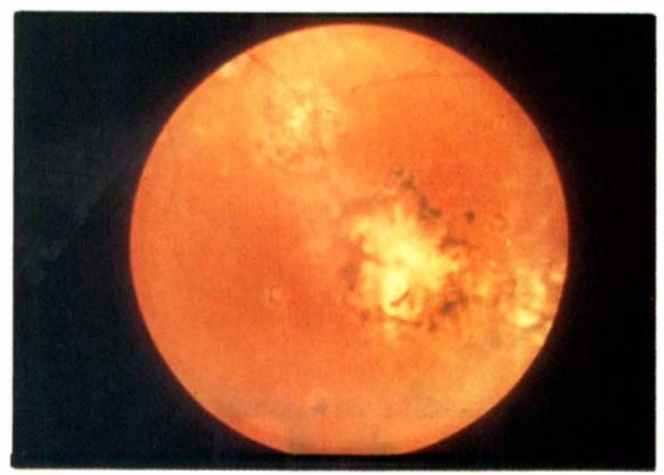

Fig. 10. Toxoplasmic scars of the retina and choroid in a 26 year-old white female. 


\section{OCULAR TOXOPLASMOSIS}

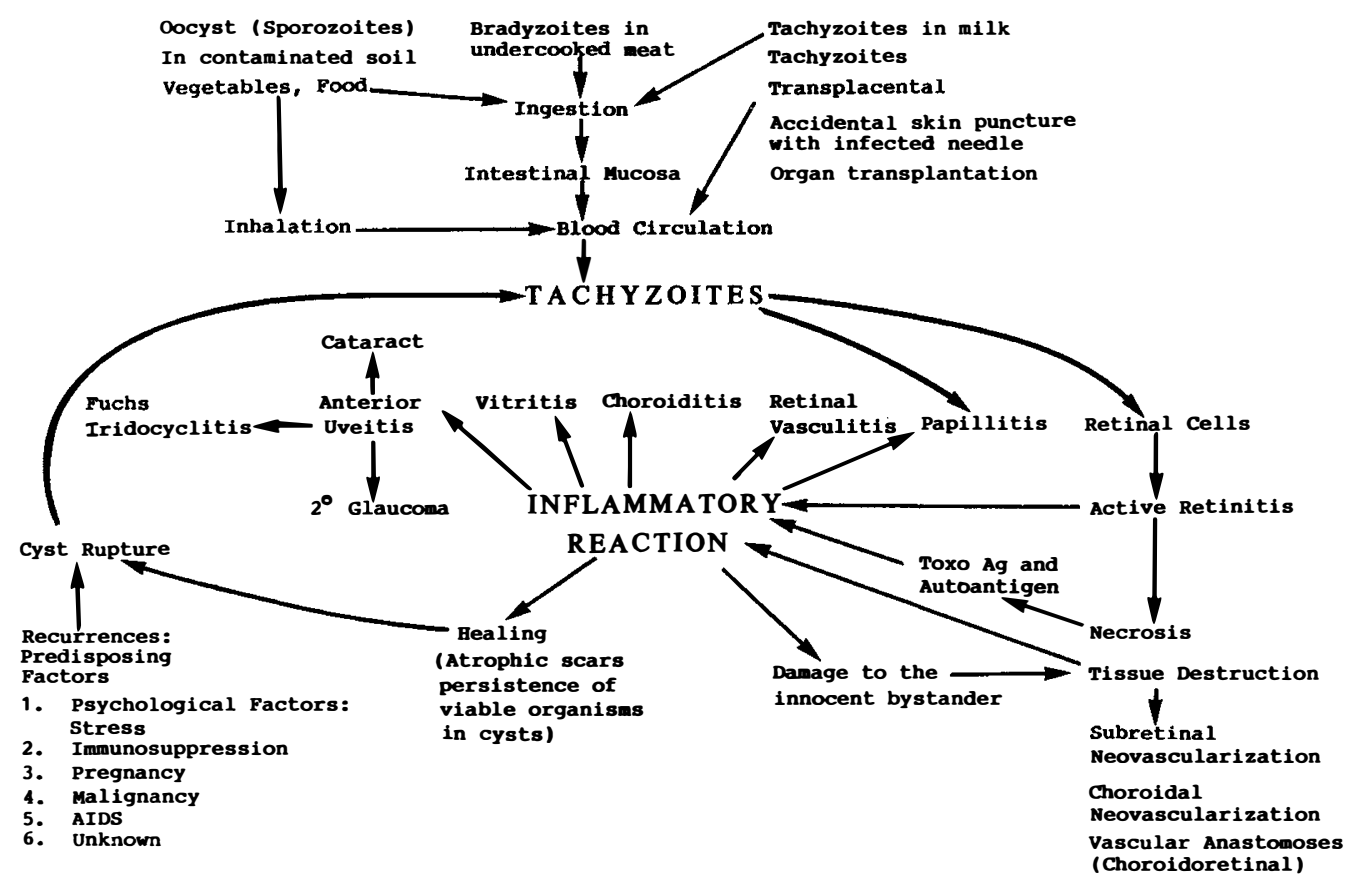

Fig. 11. Summary of the transmission and pathogenesis in ocular toxoplasmosis.

arrest the parasitic proliferation and invasion of new cells, but various products secreted by the inflammatory cells lead to further damage to the innocent bystander host cells. Infected cells may be identified by T-lymphocytes. Hypersensitivity reactions to toxoplasma antigens occur as well as to autoantigens of the photoreceptor cells..$^{13,14}$ It is apparent that we are dealing not only with toxoplasma antigens but antigens of retinal origin as well. Exaggerated cell-mediated responses to these autoantigens may be present in patients with toxoplasmic retinochoroiditis.

In patients with acquired immune deficiency syndrome (AIDS) the toxoplasmic retinochoroiditis lesion may show variation clinically and histologically. In general, ocular toxoplasmosis in AIDS patients may present with multiple and multifocal active lesions. ${ }^{15,16}$ The active lesions may occur in the absence of old healed retinochoroiditis scars but the vitreous may show evidence of inflammatory cells. Histopathologically, extensive retinal necrosis is seen in the absence of inflammatory cells. ${ }^{15}$
Toxoplasma organisms (Tachyzoites) can transform to the encysted form (Bradyzoites) under certain conditions. The cyst incorporates elements from both the host cell and the parasite itself and is, therefore, not recognised as foreign. These cysts may be tolerated for months or years without attracting any significant inflammatory reaction. ${ }^{1}$ This clever defensive mechanism is utilised by several sporozoan parasites to insure their continued existence in the tissues.

The cysts which are in the vicinity of an old scar may break down periodically due to mechanical stresses of metabolic defects. When the cyst ruptures, both viable and nonviable parasites are liberated in the adjacent tissue where viable organisms start to invade cells and cause a recurrence of the retinochoroiditis.

\section{Healing and Sequelae of Damage to the Choroidoretinal Interface:}

Resolution of the active lesions of toxoplasmic retinitis leads to a decrease in the vitreous cells. The retinal lesions become clearly 
visible with clear and sharp borders and no surrounding oedema. The deep outer retinal punctate lesions usually heal forming nummular scars. Large necrotising retinochoroiditic lesions heal with loss of the retinal and choroidal tissue in the centre of the lesions and surrounded by a retinochoroiditic scar with variable amounts of pigmentation. The tissue necrosis may induce marked pigmentary proliferation of choroidal melanocytes and retinal pigment epithelial cells leading to over-production of pigment in dark-skinned individuals and slight pigmentation in lightskinned individuals. In general marked pigmentation of retinochoroiditic scars is found in blacks and minimal pigmentation in whites. The retinochoroiditic scars in blacks appear to be heavily pigmented are more extensive than whites. There are several types of toxoplasmic retinochoroiditic scars:

(a) punched-out scar surrounded by pigment

(b) punched-out nonpigmented

(c) totally pigmented scar

(d) punctate nonpigmented

(e) punctate pigmented.

In all these scars there is loss of anatomic and functional integrity of the retina with a corresponding scotoma in the visual field. Fluorescein angiography may show late staining of the scar because of abnormal vessels in the scar. Healing of lesions next to the macular area may add insult to injury and cause contraction of the scar and macular pucker.

In large scars, neovascularisation may be seen at the choroidoretinal interface, subreinal or choroidal. Subretinal, neovascularisation in the macular area may lead to marked decrease in vision. Choroidal neovascularisation has been described by Fine et $\mathrm{al}^{17}$ as a late complication of ocular toxoplasmosis. Three patients with quiescent toxoplasmic chorioretinal scars developed choroidal neovascular membranes that led to macular detachment and sudden loss of vision. In two of the three patients the choroidal neovascularisation was subfoveal. In the third patient the choroidal neovascularisation was extrafoveal and was treated by photocoagulation.

Gaynon et al $^{18}$ described retinal neovascularisation in three patients with healed ocular toxoplasmosis associated with occlusion of a retinal arteriole or venule passing through the scar. Vascular anastomoses between the choroid and the retina may occur in patients with healed ocular toxoplasmosis as described by Doft. ${ }^{19}$ The patient had a healed retinochoroiditic scar and developed a choroidoretinal anastomosis with blood flow from a choroidal vessel (probably a choroidal artery) into a retinal vein. Vascular anastomosis between the retina and choroid has also been observed in recurrent toxoplasmosis. ${ }^{20}$ Such vascular anastomoses between the retinal and choroidal vessels occur in areas of disruption of Bruch's membrane allowing intercommunication of blood vessels within the granulation tissue that involves the retina and choroid. The flow appears to follow a resistance to flow gradient characterised by choroidal artery pressure greater than retinal artery greater than retinal vein greater than choroidal vein. Although all four possible directions of blood flow in an anastomosis between the retinal and choroidal circulation have been reported. Anastomoses include blood flow from a choroidal artery to a retinal artery ${ }^{21}$ from a retinal artery to a choroidal vein ${ }^{22}$ from a choroidal artery to a retinal vein, ${ }^{19}$ and from a retinal vein to a choroidal vein. ${ }^{20,23,24}$

\section{References}

${ }^{1}$ Tabbara KF: Toxoplasmosis. In Duane TD ed. Clinical Ophthalmology. Philadelphia. Harper and Row, 1987, 4(46): 1-23.

${ }^{2}$ Nichols B and Chiappino ML: Cytoskeleton of Toxoplasma gondii. J Protozool 1987, 34: 217-26.

${ }^{3}$ Nichols B and O'Connor GR: Penetration of mouse peritoneal macrophages by the protozoon Toxoplasma gondii: New evidence of active invasion and phagocytosis. Lab Invest 1981, 44: 324-35.

${ }^{4}$ Nichols BA, Chiappino ML, O'Connor GR: Secretion from the rhoptries of Toxoplasma gondi during host-cell invasion. J Ultrastruct Res 1983, 83: 85-98.

${ }^{5}$ Chiappino ML, Nichols BA and O'Connor GR: Scanning electron microscopy of Toxoplasma gondii: Parasite torsion and host cell responses during invasion. J Protozool 1984, 31: 288-92.

${ }^{6}$ Ryning FW and Remington JS: Effect of cytochalasin D on Toxoplasma gondii cell entry. Infect Immun 1978, 20: 739-43.

${ }^{7}$ O'Connor GR: Manifestations and management of ocular toxoplasmosis. Bull NY Acad Med 1974, 50: $192-210$.

${ }^{8}$ Nozik RA and O'Connor GR: Experimental toxoplasmic retinochoroiditis. Arch Ophthalmol 1968, 79: 485-9. 
${ }^{9}$ Tabbara KF, Nozik RA, O'Connor GR: Clindamycin effects on experimental ocular toxoplasmosis in the rabbit. Arch Ophthalmol 1974, 92: 244-7.

${ }^{10}$ Culbertson WC, Tabbara KF, O'Connor GR: Experimental ocular toxoplasmosis in primates. Arch Ophthalmol 1982, 100: 321-3.

${ }^{11}$ Doft BH and Gass JDM: Punctate outer retinal toxoplasmosis. Arch Ophthalmol 1985, 103: 1332-6.

${ }^{12}$ Rollins DF, Tabbara KF, O'Connor GR, et al: Detection of Toxoplasma antigen and antibody in ocular fluids in experimental ocular toxoplasmosis. Arch Ophthalmol 1983, 101: 455-7.

${ }^{13}$ Nussenblatt RB, Gery I, Ballintine EJ: Cellular immune responsiveness of uveitis patients to retinal S-antigen. Am J Ophthalmol 1980, 89: 173-9.

${ }^{14}$ Wyler DJ, Blackman HJ and Lunde MN: Cellular hypersensitivity to toxoplasmal and retinal antigens in patients with toxoplasmal retinochoroiditis. Am J Trop Med Hyg 1980, 29: 1181-6.

${ }^{15}$ Holland GN, Engstrom RE, Glasgow BJ, et al: Ocular toxoplasmosis in patients with the acquired immunodeficiency syndrome. Am J Ophthalmol 1988, 106: 653-67.

${ }^{16}$ Smith RE: Toxoplasmic retinochoroiditis as an emerging problem in AIDS patients. Am J Ophthalmol 1988, 106: 738-9.
${ }^{17}$ Fine SL, Owens SL, Haller JA, Knox DL, Patz A: Choroidal neovascularisation as a late complication of ocular toxoplasmosis. Am J Ophthalmol 1981, 91: 318-22.

${ }^{18}$ Gaynon MW, Boldrey EE, Strahlman ER, Fine SL: Retinal neovascularisation and ocular toxoplasmosis. Am J Ophthalmol 1984, 98: 585-9.

${ }^{19}$ Doft BH: Choroidal vascular anastomosis. Arch Ophthalmol 1983, 101: 1053-4.

${ }^{20}$ Owens PL, Goldberg MF, Busse BJ: Prospective observation of vascular anastomoses between the retina and choroid in recurrent toxoplasmosis. Am J Ophthalmol 1979, 88: 402-5.

${ }^{21}$ Braunstein RA and Gass JDM: Branch artery obstruction caused by acute toxoplasmosis. Arch Ophthalmol 1980, 98: 512-3.

${ }^{22}$ Green WR and Gass JDM: Senile disciform degeneration of the macula: Retinal arterialisation of the fibrous plaque demonstrated clinically and histopathologically. Arch Ophthalmol 1971, 86: 487-94.

${ }^{23}$ Saari M, Miettinen R, Nieminen H, et al: Retinochoroidal vascular anastomosis in toxoplasmic chorioretinitis. Acta Ophthalmol 1975, 53: 44-51.

${ }^{24}$ Kennedy JE and Wise GN: Retinochoroidal vascular anastomosis in uveitis. Am J Ophthalmol 1971, 71: 1221-5. 